\title{
Discriminating Biomolecules with Coherent Control Strategies
}

\author{
Ariana Rondia, Denis Kiseleva, Sarah Machado ${ }^{a}$, Jérôme Extermanna, Stefan Webera, \\ Luigi Bonacina ${ }^{\mathrm{a}}$, Jean-Pierre Wolfa*, Jonathan Roslund ${ }^{\mathrm{b}}$, Matthias Roth ${ }^{\mathrm{b}}$, and Herschel Rabitz ${ }^{\mathrm{b}}$
}

\begin{abstract}
The activity of the GAP-Biophotonics research group at the University of Geneva in the field of coherent control for discriminating similar biomolecules, such as flavins, proteins and DNA bases, is presented and future developments are discussed.
\end{abstract}

Keywords: Optimal Dynamic Discrimination

\section{Introduction}

The optical identification and discrimination of molecules that exhibit nearly identical absorption and fluorescence spectra is usually considered as fully elusive. However, the advent of lasers that allow time resolutions shorter than molecular motion provides new tools to reach this goal. By addressing the molecular dynamics in 'real time' and by making use of molecular quantum interference, quantum control has already exhibited its unique capability to selectively break or excite specific molecular bonds. We recently demonstrated that quantum control is able to discriminate between two nearly identical biomolecules that display almost identical absorption and fluorescence spectra: Riboflavin (RBF) and flavin mononucleotide (FMN). ${ }^{[1,2]}$ The method, called Optimal Dynamic Discrimination (ODD) is, moreover, highly sensitive, as it can use molecular fluorescence as a detection channel. ODD opens new perspectives for many laser applications, such as label-free fluorescence mi-

\footnotetext{
${ }^{*}$ Correspondence: Prof. Dr. J.-P. Wolf

Tel.: +4122 3796594

Fax: + 41223793980

E-mail: Jean-Pierre.Wolf@unige.ch

aUniversity of Geneva

GAP - Biophotonics

20, rue de l'Ecole de Médecine

$\mathrm{CH}-1211$ Genève 4

bDept. Chemistry

Princeton University

Princeton, New Jersey 08544, USA
}

croscopy, protein identification and specific detection of hazardous molecules and pathogens, also from a remote distance.

One of our major goals within the NCCR MUST is to extend ODD to more complex biological systems, such as proteins or even living organisms like bacteria.

\section{Discriminating Undistinguishable Molecules}

Our recent experiments ${ }^{[1,2]}$ aimed at testing the capabilities of ODD through the control of two structurally very similar aqueous phase flavin molecules (FMN and $\mathrm{RBF}$ ) with nearly identical linear spectroscopic features throughout the visible and into the far ultraviolet (UV) (Fig. 1a and 1b). The chemical moieties of $\mathrm{H}$ versus $\mathrm{PO}(\mathrm{OH})_{2}$ on the terminal side chains of the two flavins indirectly, and only very slightly, influence the spectroscopy of the molecules, which is mainly due to their common isoalloxazine ring structure. The laser control, determined by a closed-loop search, consists of an optimally shaped UV component at $400 \mathrm{~nm}$ (unshaped width of $80 \mathrm{fs}$ ) and a near infrared (IR) component at $800 \mathrm{~nm}$ with a Fourier transform-limited width of $100 \mathrm{fs}$, which follows the shaped UV pulse by a time-delay $\tau$ (typically 250 $500 \mathrm{fs})$.

The primary operation of ODD in this case relies on discovering the specially shaped UV pulse that coherently transfers ground state population into the flavin $S_{1}$ or $S_{2}$ excited states and amplifies the minute differences of each system's molecular Hamiltonian into resultant unique dynamical wavepackets. In the absence of the interrogating IR component, both quantum systems evolve to the same final state, detected by fluorescence, regardless of the specific UV pulse shape. However, application of the IR field disrupts the carefully created vibronic excitations of each system on a quantum mechanically coherent timescale in order to make a discriminating difference in the fluorescence signals of RBF and FMN. For both flavins, application of a IR pulse within 500 fs of excitation by an unshaped UV pulse leads to an absolute depletion of $26 \%$ in integrated fluorescence intensity. By using closed-loop optimization, multiple UV pulse shapes are discovered that in tandem with an optimally delayed (unshaped) IR pulse could modulate the relative fluorescence depletion ratio to favour either flavin. Specifically, the ratio could be varied by $\pm 28 \%$ (Fig. 1d) despite the initially indistinguishable linear and nonlinear optical spectra. Achieving this level of discrimination with nearly identical targets in the condensed phase is very impressive, especially considering that strongly decoherent processes are present. Evidence for the underlying coherence of the control process is observed by the loss of attainable dynamic discrimination for $\tau \geq 1 \mathrm{ps}$, which is a typical solution-phase coherence time. Repeated application of the closed-loop optimization technique yielded a family of uniquely shaped UV control pulses. Although this collection possesses considerable temporal variability, each member is equally successful at dynamically distinguishing the two systems. This finding is consistent with theory, which indicates that the identification of any effective control would imply the existence of many other controls of equal merit.[3] Each of these distinct control fields acts as a special interrogating photonic reagent, and the collective use of several photonic reagents allow multiplexing the determination of the fractional content of the two flavins in a micromolar mixture. The laser resources exploited for discriminating the two nearly identical flavins consisted of a modest $\sim 10$ $\mathrm{nm}$ of bandwidth centered around the 400 $\mathrm{nm}$ and $800 \mathrm{~nm}$ control pulse components. This successful demonstration of ODD 
control, even with such highly constrained fields, bodes well for future demanding quantum control applications of virtually any type aiming to draw out a desired target state in tight competition with other dynamically similar channels.

The use of the optimal pulse shapes as input parameters for $a b$ initio calculations, performed by the group of V. BonacicKoutecky, revealed the underlying mechanism of the discrimination process: ${ }^{[4]}$ the optimal laser fields drive low-frequency vibrational modes localized in the side chains of two biochromophores, thus selecting the parts of their potential energy surfaces characterized by different transition dipole moments that, in turn, lead to variable ionization probabilities.

Finally, it is worth mentioning that performance of optimal control strongly relies on the algorithm driving the closedloop experiment. We recently showed that multiobjective genetic algorithms are particularly well suited for the optimization of multiple conflicting objectives. ${ }^{[5]}$ Moreover, they represent a powerful tool to unravel the connections between pulse spectral field features and excitation dynamics of the sample.

\section{The Way to Proteins and DNA}

Many key molecules in biology, such as amino acids (tryptophan, tyrosine, phenylalanine), proteins and DNA bases have absorption bands in the deep UV (DUV), between 250 and $300 \mathrm{~nm}$. A significant problem for applying ODD to these molecules was the availability of efficient pulse shapers. Although promising results were reported with acousto-optic pulse shapers, $\left.{ }^{6} 6\right]$ significant limitations of these devices were found recently, namely two-photon absorption in the crystals and strong spatio-temporal coupling. ${ }^{[7]}$ This led us to develop a new pulse shaper based on MEMS (Micro Electro Mechanical Systems) micromirror arrays. Unlike previous developments using commercial wavefront correction devices, ${ }^{[8-10]}$ our approach consists of tailoring a micromirror array for this specific application. ${ }^{[11]}$ The 100-micromirrors contained within the device allow excellent reflectivity over the whole DUV-Vis-NIR (Al coating, Typ. 80-90\%) and negligible wavefront distortion. A novel tilt and stroke design provides, moreover, both phase and amplitude modulation of the spectral components. ${ }^{[12]}$

Transferring the ODD scheme to the DUV exhibited further difficulties: undesirable interactions with the solvent, especially at high intensities. In the strong field limit, the use of chirped pulses is probably the most direct (and intuitive)



Fig. 1. (a) Nearly identical flavin molecules: RBF and FMN exhibit almost identical absorption and fluorescence spectra (b). Coherent control-based fluorescence depletion (c) is used for efficiently discriminating the fluorescence of the two flavin molecules (d). ${ }^{[1]}$

technique for controlling vibrational coherences through intrapulse pump-dump processes. This approach was pioneered by Bardeen et al., who, back in 1995, performed a seminal experiment to show that negatively chirped pulses can be used to selectively enhance the coherent ground state population in an organic dye, while positively chirped pulses are effective at suppressing it. ${ }^{[12]}$ Given the comprehensive nature of this approach, it is very tempting to extend it to the DUV spectral region, employing tailored chirped pulses for transferring coherences between the LUMO and HOMO orbitals of biomolecules. Unfortunately, we realized that implanting such a control scheme in the UV is not suitable for addressing molecules in solution, because two-photon absorption of water and organic solvents sets in as the dominant process, hindering the effect of pulse chirp on the target molecules. More concretely, we ran a series of experiments to investigate the fluorescence efficiency of adenine and tryptophan in water as a function of the chirp of $266 \mathrm{~nm}$ pulses. For sufficient high intensities (200 GW/ $\mathrm{cm}^{2}$ per pulse), we indeed observed a deviation from the linear regime and a clear dependence of fluorescence intensity on the chirp value (i.e. larger chirp corresponding to higher fluorescence). However, this tendency was completely chirp-sign independent (Fig. 2b and 2c), contrary to what one would have expected for a pump-dump process. We later found a clear explanation for this finding by monitoring the pulse transmission through the sample. As one can see in Fig. $2 \mathrm{a}$, at sufficiently high intensities, chirped pulses are better transmitted than Fourier transform ones, as nonlinear absorption by water molecules is favoured for shorter pulses with higher peak power. Adenine fluorescence thus becomes sensitive to pulse chirp exclusively via the number of photons available for excitation through the sample.

It is worth remarking that this nonlinear interaction with solvent molecules, due to its phase-sensitive nature, sets severe limitations to the possible detection schemes applicable in coherent control experiments employing shaped UV pulses. It prevents, for example, the use of transient absorption and fluorescence as observables.[13] On the other hand, fluorescence depletion remains a sound approach: by computing the relative depletion signal $D$ for each pulse pair ${ }^{[1]} D=\left(S_{\text {und }}-S_{\text {dep }}\right) / S_{\text {und }}$ (where $S_{\text {und }}$ is the undepleted signal i.e. Without IR pulse, and $S_{\text {dep }}$ is the depleted signal, i.e. with IR pulse), any phase-dependent fluctuation of absolute fluorescence is inherently removed, isolating the molecular response from solvent-induced modulations. 

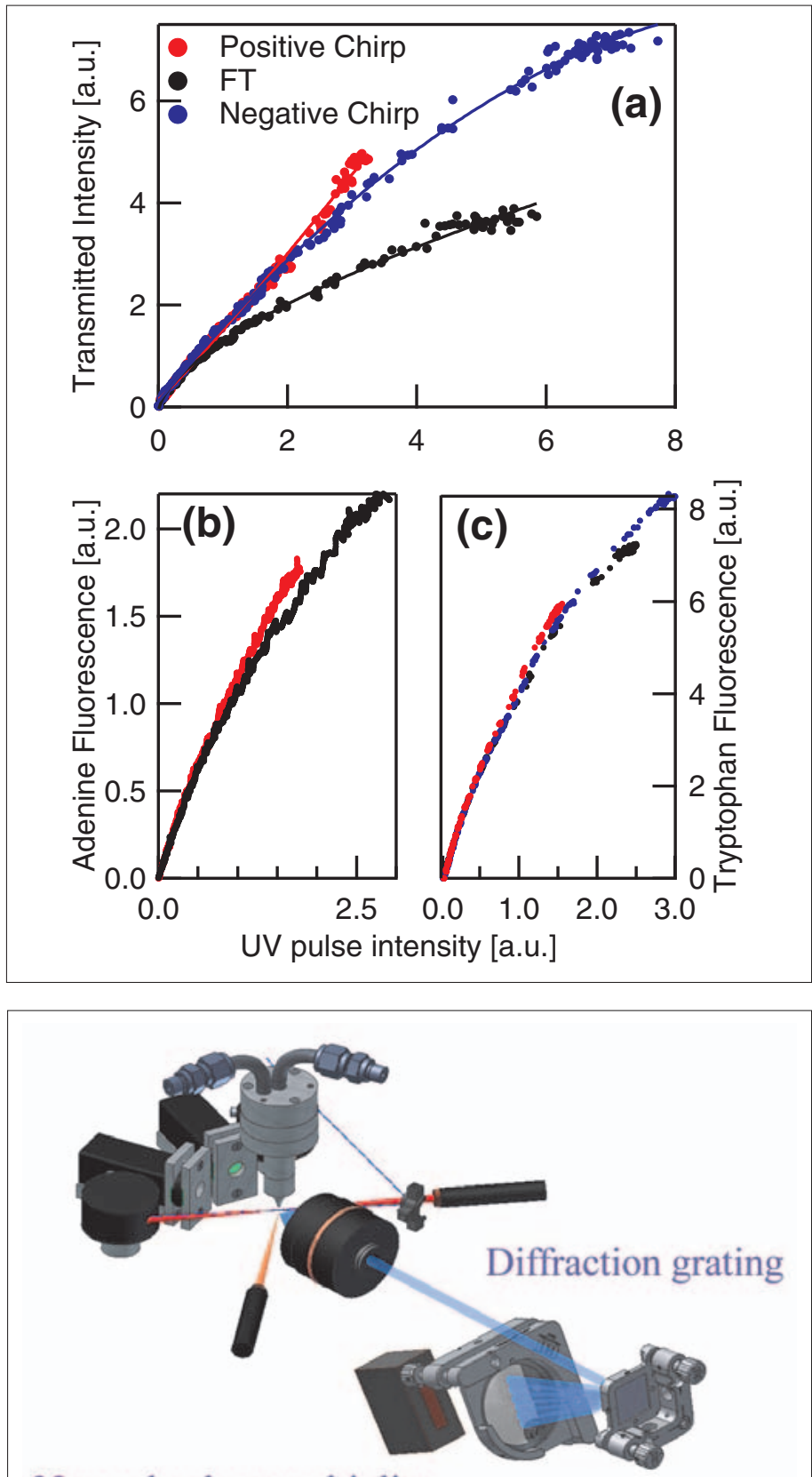

32 anode photomultiplier

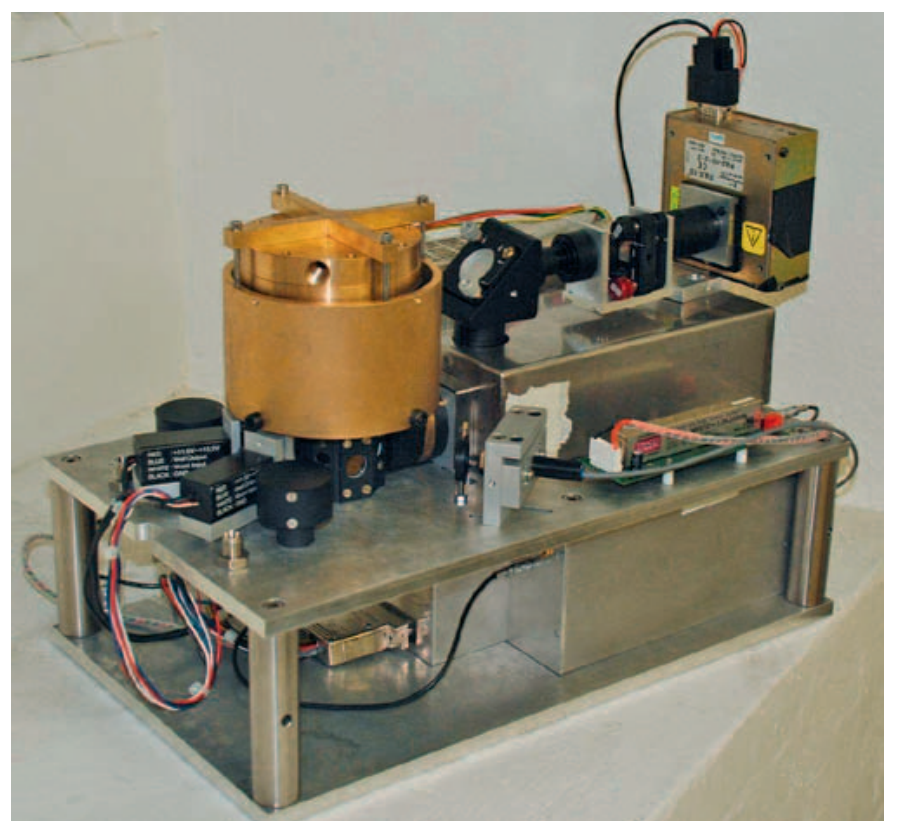

Fig. 2. (a) UV intensity transmitted by water as a function of the incident UV intensity. Lower graphs: Fluorescence of adenine (b) and tryptophan (c) as a function of incident UV intensity. The legend in (a) applies to all graphs.

Fig. 3. Aerosol analyzer. Upper panel: CAD drawing of the main parts of the device (nozzle, 2 crossed CW lasers, 2 PMTs, reflective objective, diffraction grating, 32-anode PMT). Lower panel: picture of the device in the lab.

\section{Application to Bacteria Detection}

A typical application of protein discrimination is the detection and identification of bacteria in water and air. We demonstrated a few years ago that pump-probe depletion (PPD) was able to discriminate bioaerosols from organic related aerosols, but that PPD was unable to discriminate among bacteria. ${ }^{[1,15]}$ The two major classes of bacteria, gram-positive (Staphylococcus, Bacillus, Streptococcus, Enterococcus, Listeria,...) and gram-negative (Salmonella, Escherichia coli, Yersinia pestis, Vibrio cholerae,...), are differentiated primarily because of differences in their surfaces. There are major differences between gram-positive and gram-negative bacteria in transport and attachment of the cell surface proteins. ${ }^{[16]}$ Several proteins that are periplasmic in gram-negative bacteria were observed to be lipid modified in gram-positive bacteria. ${ }^{[17]}$

Considering these striking differences between gram-positive and gram-negative bacteria, it is very appealing to perform ODD experiments for discriminating these two important classes of bacteria. Towards this goal, we developed a sampling device aimed at analyzing spectroscopically individual aerosol particles (Fig. 3).

More precisely, the apparatus designed for the generation and detection of the bioaerosols is derived from the one developed in collaboration with the group of R. K. Chang at Yale University. The bacteria are first grown in solution, and then spread as droplets of typ. $50 \mathrm{~mm}$, on demand by a piezoelectric nozzle. If the initial bacteria concentration is low enough, each microdroplet contains at most one bacterium. The droplets then pass through a thermodenuder tube, leaving the bacterium clean of water. Each of the bioaerosol particles passes through two crossed $\mathrm{CW}$ diode laser beams. The scattered light is detected by two photomultiplier tube modules.

Their signals are used to trigger the exciting laser, in our case the femtosecond laser. The fluorescence is then detected with a high aperture reflective objective, spectrally spread with a grating on a 32-anode photomultiplier tube. This set-up has provided excellent results for many elastic scattering and LIF experiments. [17-20]

A particularity of this set-up is the detection of the fluorescence in the backward direction. We indeed demonstrated that light emission from airborne particles is enhanced in the backward direction, especially if the process is nonlinear. ${ }^{[21-23]}$ Flexibility on the type of bacteria is provided by seeding the thermodenuder with two piezo-nozzles, one dedicated to the spread of the bacteria A (e.g. Gram+) and the other to bacteria B (e.g. Gram-). As the 
droplets are generated on demand, one can select which bacteria will be investigated at each event. Sequences of alternative shooting the source A or B will then provide the basis for the comparison between $\mathrm{A}$ and $\mathrm{B}$ and thus the capability of optimizing the pulse shape in a run.

\section{Conclusion and Outlook}

The ODD method, in combination with broadband pulse shapers, should allow investigation of a wealth of biological systems, from amino acids to proteins or even living organisms like bacteria. In combination with a newly developed aerosol analyzer, the novel approach offers exciting perspectives for specific detection of hazardous molecules and pathogens in water and air.

\section{Acknowledgements}

The authors would like to thank the Swiss NSF (Grant 20002020-124689), the NCCR MUST, and the University of Geneva for their continuous support.

Received: March 8, 2011

[1] M. Roth, L. Guyon, J. Roslund, V. Boutou, F. Courvoisier, J.-P. Wolf, H. Rabitz, Phys. Rev. Lett. 2009, 102, 253001.

[2] J. Roslund, M. Roth, L. Guyon, V. Boutou, F. Courvoisier, J.-P. Wolf, H. Rabitz, J. Chem. Phys. 2011, 134, 034511.

[3] T.-S. Ho, H. Rabitz, Photochem. Photobiol. 2006, 180, 226.

[4] J. Petersen, R. Mitric, V. Bonacic-Koutecky, J.P. Wolf, J. Roslund, H. Rabitz, Phys. Rev. Lett. 2010, 105, 073003.

[5] L. Bonacina, J. Extermann, A. Rondi, V. Boutou, J.-P. Wolf, Phys. Rev. A 2007, 76, 023408.
[6] N. Krebs, R. A. Probst, E. Riedle, Opt. Express 2010, 18, 6164

[7] D. J. McCabe, D. R. Austin, A. Tajalli, S. Weber, I. A. Walmsley, B. Chatel, J. Opt. Soc. Am. B 2011, 28, 58 .

[8] M. Hacker, G. Stobrawa, R. Sauerbrey, T. Buckup, M. Motzkus, M. Wildenhain, A. Gehner, Appl. Phys. B 2003, 76, 711.

[9] J. Möhring, T. Buckup, C. S. Lehmann, M. Motzkus, J. Opt. Soc. Am. B 2009, 26, 1538

[10] A. Rondi, J. Extermann, L. Bonacina, S. Weber, J.-P. Wolf, Appl. Phys. B 2009, 96, 757.

[11] J. Extermann, S. M. Weber, D. Kiselev, L. Bonacina, S. Lani, F. Jutzi, W. Noell, N. F. de Rooij, J.-P. Wolf, Opt. Express, submitted.

[12] C. Bardeen, Q. Wang, C. Shank, Phys. Rev. Lett. 1995, 75, 3410.

[13] M. Greenfield S. D. McGrane, D. S. Moore, J. Phys. Chem. A 2009, 113, 2333.

[14] F. Courvoisier, V. Boutou, V. Wood, J.-P. Wolf, A. Bartelt, M. Roth, H. Rabitz, App. Phys. Lett. 2005, 87, 063901

[15] F. Courvoisier, V. Boutou, H. Rabitz, J.-P. Wolf, J. Photochem. Photobio. A 2006, 180, 300.

[16] J. R. Scott, T. C. Barnett, Ann. Rev. Microbiol. 2006, 60, 397.

[17] W. W. Navarre, O. Schneewind, Microbio. Molec. Bio. Rev. 1999, 63, 174.

[18] S. C Hill, R. P. Pinnick, S. Niles, Y. L. Pan, S. Holler, R. K. Chang, J. Bottiger, B. T. Chen, C. S. Orr, G. Feather, Field Anal. Chem. Tech. 1999, 5, 221.

[19] Y. L. Pan, P. Cobler, S. Rhodes, A. Potter, T. Chou, S. Holler, R. K. Chang, R. G. Pinnick, J. P. Wolf, Rev. Sci. Inst. 2001, 72, 1831.

[20] Y. Pan, V. Boutou, J. R. Bottiger, S. Zhang, J.-P. Wolf, R. K. Chang, Aerosol Sci. Techn. 2004, $38,598$.

[21] S. C. Hill, V. Boutou, J. Yu, S. Ramstein, J.-P. Wolf, Y. Pan, S. Holler, R. K. Chang, Phys. Rev. Lett. 2000, 85, 54.

[22] C. Favre, V. Boutou, S. C. Hill, W. Zimmer, M. Krenz, H. Lambrecht, J. Yu, R. K. Chang, L. Woeste, J.-P. Wolf, Phys. Rev. Lett. 2002, 89, 035002.

[23] J. Kasparian, B. Krämer, J. P. Dewitz, S.Vajda, P. Rairoux, B. Vezin, V. Boutou, T. Leisner, W. Hübner, K. Bennemann, L. Wöste, J.-P. Wolf, Phys. Rev. Lett. 1997, 78, 2952. 